Praxis 15(1): 7-9

\title{
EDITORIAL
}

\section{Algunas consideraciones para el desarrollo del conocimiento pedagógico de contenido (PCK), asociado a tópicos específicos de enseñanza}

\author{
Jorge Mario Ortega Iglesias ${ }^{1}$ (D)
}

1. Editor. Universidad del Magdalena, Santa Marta, Colombia. Correo electrónico: jortegai@unimagdalena.edu.co

La práctica profesional del maestro y, especialmente, el trabajo que realiza en el aula de clases, constituyen un mundo complejo y variopinto que revela matices y singularidades epistemológicas sobre el conocimiento que este es capaz de construir en el marco de una enseñanza contextualizada, por lo que el Conocimiento Pedagógico de Contenido (PCK ${ }^{1}$ ) se ha constituido como un potente cuerpo teórico que revela ricas y variadas formas de la comprensión de cómo enseñar los contenidos constitutivos de las disciplinas escolares, particularmente, una "comprensión de lo que hace el aprendizaje de temas específicos fácil o difícil” (Shulman, 1986, p. 9).

Bajo esta potente categoría, la investigación relacionada con los procesos de caracterización del PCK ha evidenciado formas particulares y diversificadas sobre la enseñanza de las disciplinas escolares y los contenidos propios que las conforman, dicho trabajo, con amplio despliegue en las ciencias y las matemáticas, ha encontrado en el aula de clases y la práctica profesional del maestro un espacio idóneo de producción de conocimiento que ha permitido dar pasos importantes hacia la comprensión y determinación de la naturaleza del conocimiento profesional del profesorado.

Aunque caracterizar el PCK en el profesorado ha permitido evidenciar valiosos aportes para comprender la naturaleza epistemológica del conocimiento profesional del profesor, estudiar sus procesos de desarrollo precisan una apuesta de gran importancia que no debe ser descuidada (Ponte, 2012). Indagar acerca de los procesos de desarrollo del PCK, implica, por naturaleza, el descubrimiento de necesidades, oportunidades y posibilidades de mejora en la práctica cotidiana de los maestros, hecho que contribuye en la generación de nuevas formas de enseñanza y, con ello, mejores oportunidades de aprendizaje en los estudiantes.

Resulta importante comprender ampliamente los procesos de enseñanza del profesorado para avanzar en la reflexión y desarrollo del PCK con el objetivo de contribuir al mejoramiento de la práctica docente (Soine y Lumpe, 2014) y, en consecuencia, aportar en el aprendizaje de los estudiantes. Así, a continuación se presentan dos supuestos fundamentales, que consideramos son una apuesta viable, para investigar y desarrollar este tipo de conocimiento:

- En primer lugar, se declara que para desarrollar el PCK en los maestros es necesario tener en cuenta aspectos relacionados con procesos de Desarrollo Profesional Docente situado (DPDs). Algunos los elementos hacen referencia a la generación de espacios de colaboración focalizados en las experiencias de enseñanza in-situ, la articulación de intereses de enseñanza y aprendizaje producto de la colaboración, la reflexión continua y colectiva de la práctica, y, principalmente, un fuerte compromiso por el aprendizaje, tanto del sujeto que piensa la enseñanza como del sujeto que aprende de ella. Coincidimos con Van Driel y Berry (2012) en que el desarrollo profesional, bien sea como estrategia o como programa de formación docente centrado en el desarrollo del PCK, no pueden limitarse a brindar

\footnotetext{
${ }^{1}$ Traducción del inglés Pedagogical Content Knowledge
} 
Algunas consideraciones para el desarrollo del conocimiento pedagógico de contenido (PCK), asociado a tópicos específicos de enseñanza

"información" como ejemplos de enseñanza de expertos en la materia, por el contrario, tales programas deben estar estrechamente relacionados con la práctica profesional de los docentes y, además, generar oportunidades de cambio en donde la reflexión colectiva sobre las estrategias de enseñanza y aprendizaje de contenidos específicos sea una experiencia de aprendizaje continuo.

- En segundo lugar, se asume que una vía legítima y contextualizada para desarrollar el PCK sobre tópicos específicos de las disciplinas escolares, obedece a su trabajo en el marco de Comunidades Profesionales de Aprendizaje (CPA) con un enfoque práctico y reflexivo. Existe cierta preocupación sobre los intereses y prioridades identificados al interior de las comunidades de profesores, en las que se ignoran los problemas relacionados con la enseñanza y aprendizaje de las disciplinas escolares (Van Driel y Berry, 2012; Bausmith y Barry, 2011) y, más específicamente, los problemas para enseñar y aprender los contenidos específicos. La idea fuerte que moviliza el trabajo, en el marco de una CPA, es que el conocimiento que emerge de este contexto es el resultado de "una construcción social compartida" (Louis, 2006, p. 480).

Los caminos empleados para el desarrollo del PCK centrado en tópicos o contenidos específicos de las disciplinas escolares, componen un pilar para el diseño de estrategias de desarrollo profesional docente situado que promueven la mejora educativa desde el interior del aula de clases (Duque, Celis, Díaz, Gómez, 2014); su implementación requiere superar una visión y actuación docente aislada, reservada, exclusiva entre el profesor y los estudiantes, y pocas veces compartida con los colegas que acompañan la labor docente (Shulman, 1993; Bolívar, 2017). En este sentido, desarrollar el PCK constituye un reto de colaboración y articulación colectiva de intereses, que haga visible lo invisible de la experiencia de enseñar, pero que, principalmente, constituya una oportunidad de aprendizaje colectivo en el que se beneficien profesores, estudiantes y comunidad en general.

Estas aproximaciones argumentativas nos proponen el desarrollo de algunos interrogantes necesarios para abonar el camino teórico que soporte el proceso coherente de desarrollo del PCK. Así, vale la pena preguntarse: ¿Cómo se relacionan las categorías PCK y DPDs?, ¿Cuáles son los contenidos disciplinares a priorizar en la caracterización del PCK, para una mejora en las prácticas de aula de un colectivo de profesores?, ¿Cómo se articulan las categorías PCK y CPA?, ¿De qué manera la implementación de una CPA promueve el desarrollo del PCK en el profesorado?, ¿Cuáles son las rutas metodológicas que permitirían la integración de estas categorías?

Estos elementos constituyen un pretexto académico para dar apertura a un escenario de investigación que nos motive a profundizar en procesos de desarrollo profesional docente situado, puntualmente en aquellos donde la enseñanza en el aula, la colaboración entre profesores, la reflexión colectiva de la enseñanza y el desarrollo del PCK para promover el aprendizaje sean una prioridad. Por lo tanto, las consideraciones que aquí se presentan permitirán abrir un campo de oportunidades investigativas movilizado por el siguiente eje problematizador: ¿Cómo explicitar y desarrollar el conocimiento pedagógico de contenido de un tópico específico, en el marco de una comunidad profesional de aprendizaje?

\section{Referencias Bibliográficas}

Bausmith, J. M. y Barry, C. (2011). Revisiting Professional Learning Communities to Increase College Readiness: The Importance of Pedagogical Content Knowledge. Educational Researcher, 40(4), 175-178. Doi: 10.3102/0013189X11409927

Bolívar, A. (2017). Investigación y docencia: de una relación problemática a una productiva. Aula Magna 2.0. [Blog]. Recuperado de https://cuedespyd.hypotheses.org/2641

Duque, M., Celis, J., Díaz, B. y Gómez, M. (2014). Diez pilares para un programa de desarrollo profesional docente centrado en el aprendizaje de los estudiantes. Revista Colombiana De Educación, 1(67), 107-124. Doi: 10.17227/0120391.67rce107.124

Gordon, S. P. (2006). Professional Development for School Improvement: Empowering Learning Communities. Boston, MA: Allyn and Bacon.

Hargreaves, A. (2008). Leading professional learning communities: Moral choices amid murky realities. En A. M. Blankstein, 
P. D. Houston y R. W. Cole (Eds.), Sustaining professional learning communities (pp. 175-197). Thousand Oaks, CA: Corwin Press.

Louis, K. S. (2006). Changing the culture of schools: Professional community, organizational learning, and trust. Journal of School Leadership, 16(5), 477-489.

Shulman, L. (1989). Paradigmas y programas de investigación en el estudio de la enseñanza: Una perspectiva contemporánea. En M. Wittrock (Ed.). La investigación en la enseñanza, I. Enfoques, teorías y métodos (pp. 9-91). Madrid, España: Paidos-MEC.

Shulman, L. (1993). Teaching as community property: Putting an end to pedagogical solitude. Re-search Library, 25(6), 6-7.

Soine, K. M. y Lumpe, A. (2014). Measuring characteristics of teacher professional development. Teacher Development, 18(3), 303-333. Doi: 10.1080/13664530.2014.91 1775

Valliant, D. (2016). Trabajo colaborativo y nuevos escenarios para el desarrollo profesional docente. Revista Hacia Un Movimiento Pedagógico Nacional, 60, 07-13.

Van Driel, J. y Berry, A. (2012). Teacher Professional Development Focusing on Pedagogical Content Knowledge. Educational Researcher, 41(1), 26-28. Recuperado de http://www.jstor.org.biblioteca.unimagdalena.edu.co:2048/stable/41413082 